\title{
Lemierre's syndrome due to Klebsiella pneumoniae in a 63-year-old man with diabetes: a case report
}

\author{
Musa A Garbati', Azeem M Ahsan and Ahmed M Hakawi
}

\begin{abstract}
Introduction: Lemierre's syndrome was originally documented to be caused by Fusobacterium necrophorum. It is a very rare condition with a prevalence of one to 14.4 instances per million. Its presentation is varied, not only in composition but also in the infecting organism. Treatment with anticoagulants has been controversial and applied only on a case-by-case basis.

Case presentation: A 63-year-old Saudi man who had had uncontrolled diabetes mellitus for 47 years presented to our facility with a five-day history of swelling on the right side of his neck and fever. The swelling progressively increased in size and was associated with pain, dysphagia, odynophagia, change of voice ('hot potato voice'), and reduced appetite. Abscess content culture and sensitivity testing revealed Klebsiella pneumoniae. However, blood culture results were repeatedly negative. The abscess was incised and drained without any complication. Our patient was treated with clindamycin and cefuroxime. Warfarin was also administered concurrently for six weeks, for an isolated internal jugular vein thrombosis (IJV), with complete resolution of the thrombus. Normoglycemia was achieved and our patient was discharged after complete wound healing and the return of his biochemical parameters to normal.

Conclusions: Only two cases of Lemierre's syndrome in patients with diabetes due to K. pneumoniae have been reported previously. A review of the literature suggested that an association exists between deep neck infections due to $K$. pneumoniae and diabetes mellitus. The reasons for this association are still not clear. This poses a question as to whether diabetes mellitus specifically predisposes these patients to infection with this organism. It is suggested that clinicians should consider infectious agents other than F. necrophorum in the causation of Lemierre's syndrome, especially in patients with diabetes.
\end{abstract}

\section{Introduction}

Post-anginal sepsis, otherwise known as Lemierre's syndrome (LS) or necrobacillosis, was first reported by Courmont and Cade in 1900 [1]. The condition was better described by Andre Lemierre in 1936 [2] from a report of 20 cases. The hallmark of the syndrome, as was originally described by Lemierre, is a tetrad of acute oropharyngeal infection, septicemia, thrombophlebitis of the ipsilateral internal jugular vein (IJV), and secondary metastatic abscesses, most commonly to the lungs and joints. The syndrome has mainly been described in young, immunocompetent individuals, with a prevalence

\footnotetext{
* Correspondence: musagarbati@yahoo.com

Division of Infectious Diseases, Department of Medicine, King Fahad Medical City, 11525, Riyadh, Saudi Arabia
}

of one to 14.4 instances per million. This condition therefore requires a high index of suspicion for diagnosis [3]. The initiating infection is usually due to a Gramnegative anaerobe, $F$. necrophorum (previously known as Bacillus fundiliformis), but other organisms have also been implicated. Occasionally, no organisms were grown from clinical specimens [4].

LS was reported to be usually fatal prior to the discovery of antibiotics, with up to $90 \%$ mortality. The introduction of penicillin in the 1940s for oropharyngeal infections led to a significant drop in the incidence of the disease and its associated mortality (to as low as $5 \%$ ) [5]. This has led to the disease becoming forgotten [6] and also to the appearance of incomplete forms of the syndrome.

\section{Biomed Central}


We describe a rare case of LS in a patient with diabetes caused by K. pneumoniae. We also review the relevant literature.

\section{Case presentation}

In November 2010, a 63-year-old Saudi man with background diagnoses of bronchial asthma and type 1 diabetes mellitus (DM I) of 47 years, presented to our facility with a five-day history of swelling on the right side of his neck and fever. The swelling progressively increased in size and was associated with pain, dysphagia, odynophagia, change of voice ('hot potato voice'), and reduced appetite. There was no prior history of shortness of breath, upper respiratory tract infection (URTI), dental problems or procedures. On examination our patient was found to be febrile $\left(38.8^{\circ} \mathrm{C}\right)$, diaphoretic with a pulse rate of 115 beats per minute, a respiratory rate of 20 cycles per minute, BP of $119 / 69 \mathrm{mmHg}$ and oxygen saturation of $99 \%$ at room air. There was a right submandibular swelling $(5 \times 5 \mathrm{~cm})$ with all the cardinal features of acute inflammation (rubor, calor, tumor, dolor, and functio laesa). The first four of these signs were named by Celsus in ancient Rome (30-38 B.C.) and the last by Galen (A.D 130-200) [7].The right tonsil was enlarged and the uvula medially displaced.
Examination of the respiratory system did not reveal any abnormalities.

Laboratory investigations revealed a white cell count of $10.5 \times 10^{9}$ cells/L (neutrophils $80.8 \%$ ), hemoglobin $13.9 \mathrm{~g} / \mathrm{dL}$, platelet count $479 \times 10^{9}$ cells/L, erythrocyte sedimentation rate (ESR) $44 \mathrm{~mm} /$ hour and international normalized ratio (INR) of 3.1. Serum glucose was 23 $\mathrm{mmol} / \mathrm{L}$, total cholesterol $2.9 \mathrm{mmol} / \mathrm{L}$, low-density lipoprotein (LDL) cholesterol $1.79 \mathrm{mmol} / \mathrm{L}$, high-density lipoprotein (HDL) cholesterol $0.5 \mathrm{mmol} / \mathrm{L}$ and triglyceride $1.42 \mathrm{mmol} / \mathrm{L}$. Hemoglobin A1c (HbA1c) was 9.9\% on admission. Alanine aminotransferase (ALT) was 21 $\mathrm{U} / \mathrm{L}$, aspartate aminotransferase (AST) $6 \mathrm{U} / \mathrm{L}$, uric acid $246 \mu \mathrm{mol} / \mathrm{L}$, urea $5.5 \mathrm{mmol} / \mathrm{L}$, creatinine $90 \mu \mathrm{mol} / \mathrm{L}$, and total bilirubin $10.5 \mu \mathrm{mol} / \mathrm{L}$. Abscess content culture and sensitivity testing yielded $K$. pneumoniae; however, blood culture results were negative. Serotyping of the $K$. pneumonia isolate from our patient was not performed.

A computed tomography $(\mathrm{CT})$ scan (Figure 1) of the neck region showed a multiloculated abscess (circle of arrowheads) with irregular peripheral enhancement in the right carotid space extending from the second to the fourth cervical vertebrae. The right sternomastoid muscle was swollen with surrounding inflammatory changes as well as multiple enlarged cervical lymph nodes. The

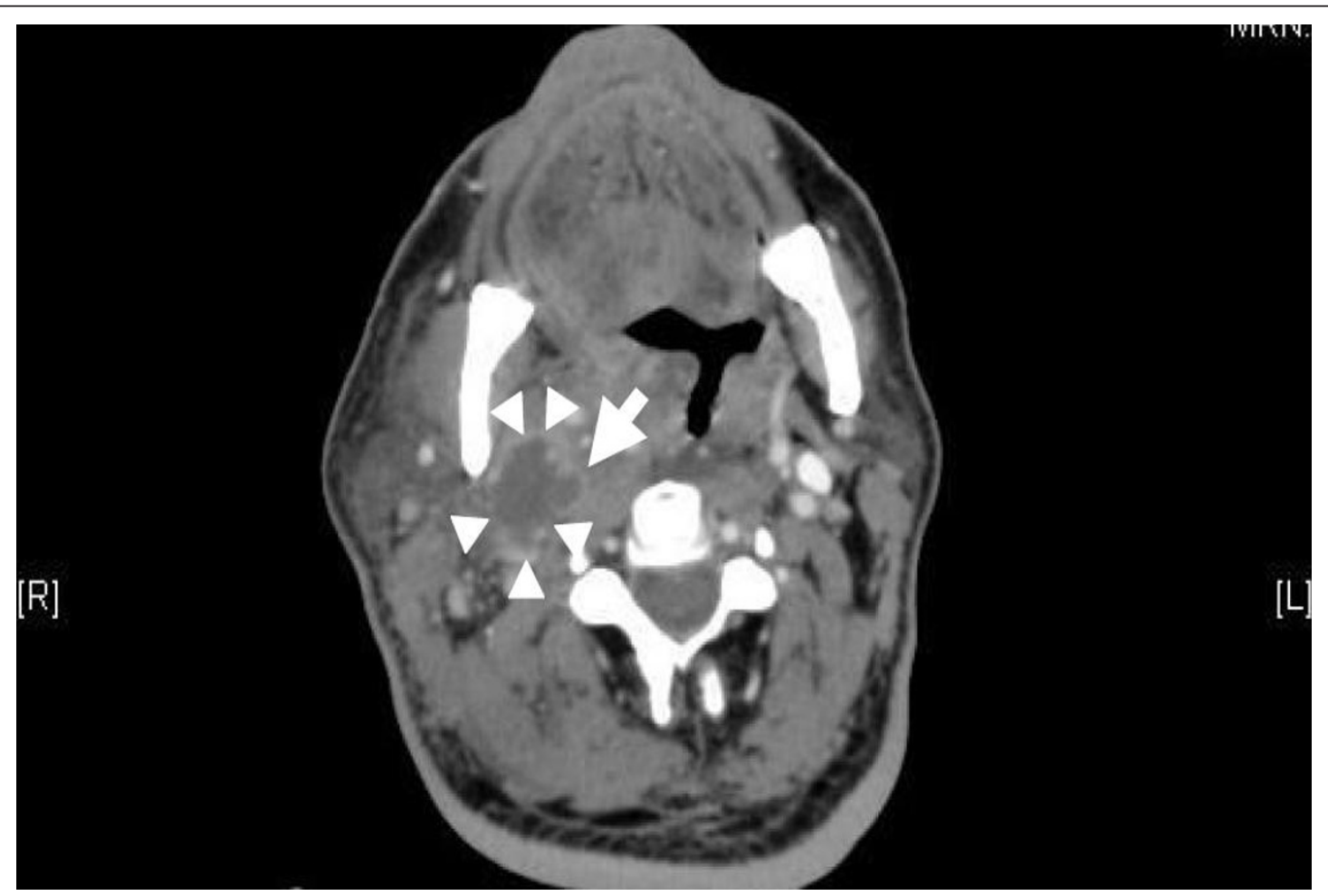

Figure 1 Computed tomography (CT) scan of the neck region showing the abscess content (circle of arrowheads) and thrombosis in the right internal jugular vein (single arrow). 
fluid tracked through the pre-vertebral space from the $\mathrm{C} 2$ down to the $\mathrm{C} 6$ level, with a maximum anteroposterior (AP) diameter of $7 \mathrm{~mm}$. It tracked into the right parapharyngeal space down to the supraglottic region. The palatine tonsils were enlarged, more significantly on the right side, and showed small areas of low attenuation. The right internal jugular vein (IJV) showed thrombosis and enhancement of its walls (single arrow in Figure 1) that extended intracranially into the right sigmoid and transverse venous sinuses. The thrombus also extended inferiorly down to the $\mathrm{C} 7$ level. Other features noted on the CT included a mass effect on the oropharynx and hypopharynx as well as the larynx. A CT scan of the chest was normal.

Our patient underwent incision and drainage of the abscess with no complications. He was treated with a combination of clindamycin and cefuroxime in addition to warfarin for six weeks. These interventions resulted in complete resolution of the thrombus. Normoglycemia was achieved before his discharge from hospital, and subsequent follow-up was uneventful. He was finally discharged from the clinic after complete wound healing and the return of his biochemical parameters to normal levels.

\section{Discussion}

Historically, LS has been reported to most commonly affect young, healthy adults, although cases in children have also been reported [8]. Riordan recently reviewed 222 cases of LS and reported that in up to $89 \%$ the diagnosis was in individuals aged 10 to 35 years [9]. Since its initial description the condition has gone from being highly fatal, in the pre-antibiotic era, to a curable disease with the use of potent antimicrobials. The classic tetrad of features reported earlier in the century has given way to variants or incomplete forms of the disease with extension of the affected age group and variation in its microbiology. This has brought to the fore variants of or Lemierre-like syndromes, either due to isolation of organisms other than F. necrophorum [9] or presentations that were short of the tetrad originally described or due to the involvement of anatomic structures other than those reported earlier. A retrospective review of peri-tonsillar abscesses from Saudi Arabia between 2000 and 2004 by Al-Dakhail and Khan [10] revealed that out of the 65 samples sent for culture and sensitivity, 29.6\%, $21 \%$, and $3.7 \%$ yielded normal flora, group A Streptococci, and Staphylococcus aureus, respectively; while $K$. pneumoniae and Streptococcus viridians constituted $2.5 \%$ each of the isolates. No cases of F. necrophorum infection were reported in their patients. Some authors have questioned whether the isolation of $F$. necrophorum is really necessary for the diagnosis of LS [11]. Indeed a recent review reported $6 \%$ to $14 \%$ of cases of LS were associated with either negative cultures or organisms other than F. necrophorum [9]. It is of interest to note that the isolation of $F$. necrophorum requires strict anaerobic culture conditions. Other reasons that could have possibly led to negative blood cultures in our patient is prior antibiotic use or that $F$. necrophorum was overlooked, particularly by laboratory staff unfamiliar with its typical features and characteristics.

Involvement of $K$. pneumoniae in the causation of LS is rare, with only two previously reported cases [11,12] both of whom were also diabetic; therefore the question arose as to whether diabetes mellitus (DM) had a role in selecting this organism, or it was a mere coincidence? This association has been reported previously by Huang et al. [13], where $98.4 \%$ of their patients with diabetes had infections due to K. pneumoniae. A recent review from Singapore [14] by Lee and Kanagalingam documented about $50 \%$ of their patients with diabetes with deep neck abscesses had K. pneumoniae isolated from the collections. Some of these abscesses were found to be caused by the hypermucoviscosity (HV) phenotype of K. pneumoniae, especially $\mathrm{K} 1$ and $\mathrm{K} 2$ isolates which tend to be significantly more resistant to phagocytosis than non-K1/K2 isolates [15].

Like other Gram-negative bacteria, F. necrophorum produces lipopolysaccharide, which behaves like a classical endotoxin with virulence in many animal models [9]. Development of the disease has been described to occur in three stages: (1) infection of deep pharyngeal tissue; (2) invasion of the lateral pharyngeal space, resulting in IJV thrombophlebitis; and (3) embolic spread of the infection. The exact etiology and pathogenesis of LS is still unknown, but lymphatic or direct spread of infection from the thrombophlebitis of the tonsillar veins causing thrombosis of the IJV due to direct involvement of the alveolar tissue of the neck has been suggested as a possible mechanism [16].

In the absence of controlled trials to determine the optimum therapy for LS, most authors based their choice of antimicrobial agent(s) mainly on personal experience and anecdotal evidence. Metronidazole is usually the most preferred agent though some authors used either a carbapenem or a penicillin/ $\beta$-lactamase inhibitor combination for a total of six weeks, but the optimal duration of treatment is not known.

The use of anticoagulants has been controversial, as no controlled trials exist. Some authors observed persistence of fever, worsening clinical condition and ongoing radiographic evidence of pulmonary emboli until full anticoagulant therapy has been initiated [17]. Routine anticoagulation is not generally required for all cases of IJV thrombosis in the absence of convincing data from randomized studies. However, anticoagulation has been frequently advocated in situations where untoward 
consequences are expected such as the involvement of the sigmoid or cavernous sinuses. Another indication for anticoagulation in patients with LS would be suppurating thrombophlebitis of the sigmoid sinus propagating to the cavernous sinus.

\section{Conclusions}

Cases of LS are rare. It may be caused by organisms not originally described by Andre Lemierre. Clinicians should therefore look out for organisms other than $F$. necrophorum when clinical suspicion is high and also broaden the antimicrobial cover. In all cases reported due to $K$. pneumoniae, the patients happen to be diabetic; therefore the question arose as to whether diabetes mellitus specifically selects for this organism. Available evidence suggests this could be the case. The role of anticoagulation has been controversial and since no randomized trials are available, this approach has only been applied on a case-by-case basis. Accurate and timely diagnosis is critical because untreated disease is usually fatal. Primary care providers should therefore be aware of the syndrome in patients with oropharyngeal infection who subsequently show signs of systemic illness or pulmonary involvement.

\section{Consent}

Written informed consent was obtained from the patient for publication of this case report and any accompanying images. A copy of the written consent is available for review by the Editor-in-Chief of this journal.

\section{Authors' contributions}

MAG designed and prepared the manuscript. AMA managed our patient and also reviewed the manuscript. $\mathrm{AMH}$ reviewed the manuscript. All authors have read and approved the final manuscript for publication.

\section{Competing interests}

The authors declare that they have no competing interests.

Received: 19 September 2011 Accepted: 3 April 2012

Published: 3 April 2012

\section{References}

1. Courmont P, Cade A: Sur une septico-pyohémiede l'homme simulant la peste et causée par un streptobacille anaérobie. Arch Méd Exp Anat Pathol 1900, 4.

2. Lemierre A: On certain septicemias due to anaerobic organisms. Lancet 1936, 1:701-703.

3. Ajulo P, Qayyum A, Brewis C, Innes A: Lemierre's syndrome: the link between a simple sore throat, sore neck, and pleuritic chest pain. Ann $R$ Coll Surg Engl 2005, 87:303-305.

4. Chirinos JA, Lichtstein D, Garcia J, Tamariz LJ: The evolution of Lemierre syndrome: report of 2 cases and review of the literature. Medicine (Baltimore) 2002, 81:458-465.

5. Karkos PD, Asrani S, Karkos CD, Leong SC, Theochari EG, Alexopoulou TD, Assimakopoulos AD: Lemierre's syndrome: a systematic review. Laryngoscope 2009, 119:1552-1559.

6. Shah SA, Ghani R: Lemierre's syndrome: a forgotten complication of oropharyngeal infection. J Ayub Med Coll Abottabad 2005, 17:30-33.
7. Hurley JV: Acute inflammation. Edinburgh, London: Churchill Livingstone; 1972.

8. Schmid T, Miskin H, Schlesinger Y, Argaman Z, Kleid D: Respiratory failure and hypercoagulability in a toddler with Lemierre's syndrome. Pediatrics 2005, 115:e620-e622.

9. Riordan T: Human infection with Fusobacterium necrophorum (necrobacillosis), with a focus on Lemierre's syndrome. Clin Microbiol Rev 2007, 4:622-659.

10. Al-Dakhail AA, Khan Ml: A retrospective study of peritonsillar abscess in Riyadh Medical Complex toward setting up a treatment protocol. Saudi Med J 2006, 27:1217-1221.

11. Singaporewalla RM, Clarke MJ, Krishnan PU, Tan DEL: Is this a variant of Lemierre's syndrome? Singapore Med J 2006, 47:1092-1095.

12. Bhgat B, Ahmed M, Ali SW, Roistacher K: A case of Lemierre's syndrome due to Klebsiella pneumonia. Infect Dis Clin Pract 1996, 5:389-391.

13. Huang $T$, Liu TC, Chen PR, Tseng FY, Yeh TH, Chen YS: Deep neck infection: analysis of 185 cases. Head Neck 2004, 26:854-860.

14. Lee $Y Q$, Kanagalingam J: Deep neck abscesses: the Singapore experience. Eur Arch Otorhinolaryngol 2011, 268:609-614.

15. Lin JC, Chang FY, Fung CP, Xu JZ, Cheng HP, Wang JJ, Huang LY, Siu LK: High prevalence of phagocytic-resistant capsular serotypes of Klebsiella pneumoniae in liver abscess. Microbes Infect 2004, 6:1191-1198.

16. Yau PC, Norante JD: Thrombophlebitis of the internal jugular vein secondary to pharyngitis. Arch Otolaryngol 1980, 106:507-508.

17. Razonable RR, Rahman AE, Wilson WR: Lemierre syndrome variant: necrobacillosis associated with inferior vena cava thrombosis and pulmonary abscesses after trauma-induced leg abscess. Mayo Clin Proc 2003, 78:1153-1156.

doi:10.1186/1752-1947-6-97

Cite this article as: Garbati et al:: Lemierre's syndrome due to Klebsiella pneumoniae in a 63-year-old man with diabetes: a case report. Journal of Medical Case Reports 2012 6:97.

\section{Submit your next manuscript to BioMed Central and take full advantage of:}

- Convenient online submission

- Thorough peer review

- No space constraints or color figure charges

- Immediate publication on acceptance

- Inclusion in PubMed, CAS, Scopus and Google Scholar

- Research which is freely available for redistribution 